\title{
A Study of Antibacterial Activity of Some Novel 8-Methoxy-4-methyl-quinoline Derivatives
}

\author{
Sheoraj Singh, Vikas Kumar, Ashok Kumar, Shalabh Sharma, ${ }^{\dagger, *}$ and Piyush Dua \\ Medicinal Chemistry Division, Department of Pharmacology, L. L. R. M. Medical College, Meerut-250 004 (U.P.), India \\ ${ }^{\dagger}$ Department of Chemistry, D. J. College of Engineering \& Technology, Niwari Road, Modinagar-201 204 (U.P.), India \\ "E-mail: drshalabhsharma@gmail.com \\ ${ }^{\ddagger}$ Centre for Superfunctional Materials, Pohang University of Science and Technology, San 31, Hyoja-dong, Namgu, \\ Pohang 790-784, Korea
}

Received April 12, 2010, Accepted September 29, 2010

\begin{abstract}
In the present study, some quinoline derivatives have been synthesized like 8-methoxy-4-methyl-2-amino-(3'-chloro2'-oxo-4'-substituted aryl-1'-azetidinyl)quinolines 8-12 and 8-methoxy-4-methyl-2-amino-(2'-substituted aryl-4'-oxo1',3'-thiazolidin-3'-yl) quinolines 13-17 from 8-methoxy-4-methyl-2-(substituted arylidenyliminoamino)-quinolines 3-7. The structural assignments of these compounds were based on spectral (IR, ${ }^{1} \mathrm{H}-\mathrm{NMR}$, Mass) and elemental (C, $\mathrm{H}$, $\mathrm{N}$ ) analysis. Further, these compounds were evaluated for antibacterial activity against various bacterial strains. Three compounds 10,11 and 16 were found to exhibit potent antibacterial activity as compared to the standard drug amphicillin.
\end{abstract}

Key Words: Quinoline, Schiff base, Azetidinone, Thiazolidinone, Antibacterial activity

\section{Introduction}

To treat the infectious diseases caused by various bacteria is still remain challenging due to the increasing number of drug resistant microbial pathogens, this in turn unable the drugs to control the growth of harmful microorganisms. By considering this fact, it was suggested to employ structurally novel therapeutic agents with enhanced broad spectrum of potency against increasingly resistant pathogens. ${ }^{1}$ Therefore, there is a need to explore some novel antibacterial agents. The recent decades have seen that medicinal chemists have devoted significant efforts to develop various derivatives of quinoline as antibacterial agents. Literature survey has indicated that quinoline derivatives possess diverse pharmacological activities like antimicrobial, ${ }^{2}$ anti-malarial, ${ }^{3}$ antiviral, ${ }^{4}$ anti-tumor, ${ }^{5}$ immunomodulatory, ${ }^{6}$ antileishmanial, ${ }^{7}$ anti-inflammatory activity, ${ }^{8}$ and many more. The congeners of schiff base, ${ }^{9-10}$ azetidinone ${ }^{11-12}$ and thiazolidinone ${ }^{13-14}$ have also been proved to exhibit promising antibacterial activity. These finding prompted us to synthesize the substituted quinoline derivatives by the combination of azetidinone or thiazolidinone moieties in one frame, this may lead to the compounds with interesting antibacterial profile.

\section{Chemistry}

The starting material 8-methoxy-4-methyl-2-chloroquinoline 1 was prepared by 8-methoxy-4-methyl-quinolin-2-ol on reaction with phosphorous oxychloride. This compound was reacted with hydrazine hydrate to furnish hydrazide congener: 8methoxy-4-methyl-2-hyrazinoquinoline 2 , which on reaction with numerous aromatic aldehydes in presence of glacial acetic acid to yield schiff bases: 8-methoxy-4-methyl-2-(substituted arylidenyliminoamino)-quinolines 3-7. Compounds 3-7 were treated with chloroacetyl chloride and triethylamine to produce azetidinones: 8-methoxy-4-methyl-2-amino-(3'-chloro- 2'-oxo-4'-substituted aryl-1'-azetidinyl)quinolines 8-12. On the other hand, substituted thiazolidinones: 8-methoxy-4-methyl-2amino-(2'-substituted aryl-4'-oxo-1',3'-thiazolidin-3'-yl)quino-

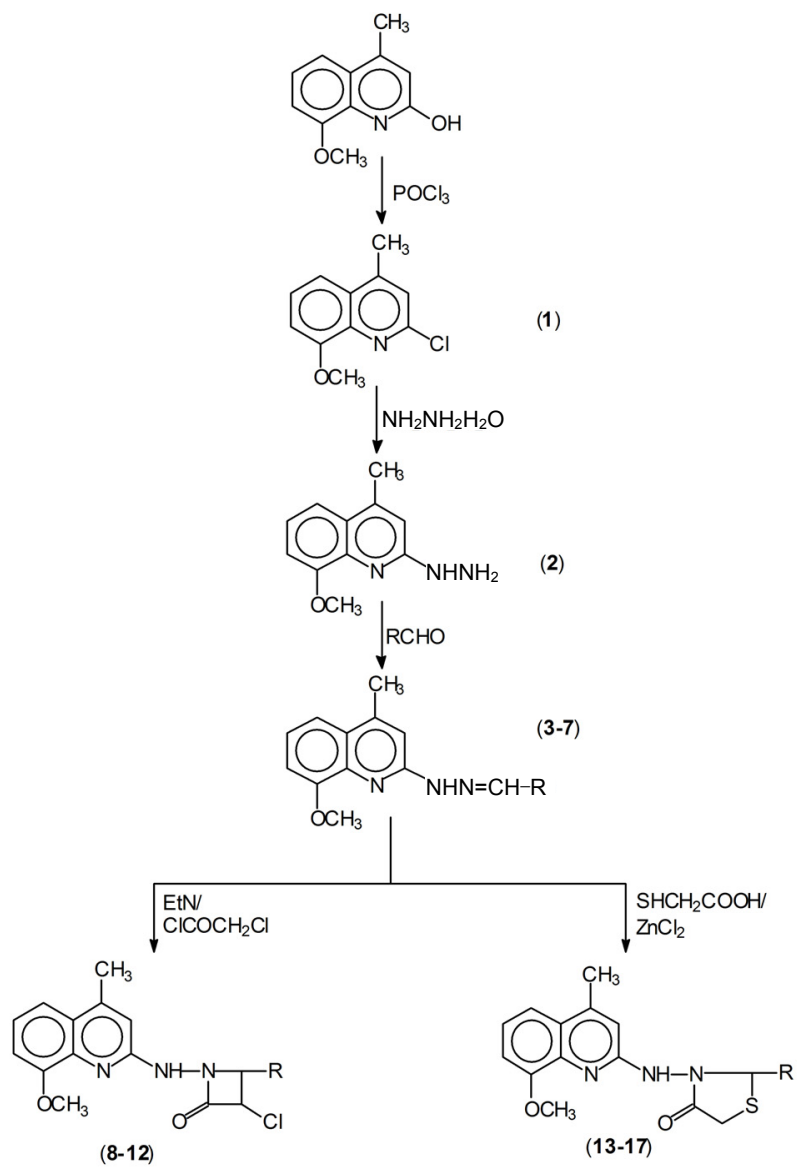

Scheme 1 
lines 13-17 have been procured from compounds 3-7 on reaction with thioglycolic acid and zinc chloride. The synthetic route of above said compounds is shown in Scheme 1.

\section{Results and Discussion}

With the aim to develop new antibacterial agents, we have reported the synthesis of 8-methoxy-4-methyl-2-(substitutedarylidenyliminoamino)-quinolines 3-7, 8-methoxy-4-methyl2-amino-(3'-chloro-2'-oxo-4'-substituted aryl-1'-azetidinyl)quinolines 8-12 and 8-methoxy-4-methyl-2-amino-(2'-substitutedaryl-4'-oxo-1',3'-thiazolidin-3'-yl) quinolines 13-17. Then, these compounds were screened in vitro for antibacterial activity against gram positive bacteria: Staphylococcus aureus ATCC 25923 (S. aureus), Bacillus subtilis ATCC 6051 (B. subtilis) and Staphylococcus epidermis ATCC 14940 (S. epidermis) and gram negative bacteria Escherichia coli ATCC 25922 ( $E$. coli), Klebsiella pneumoniae ATCC 10031 (K. pneumoniae) and Pseudomonas aeruginosa ATCC 27853 (P. aeruginosa) at a concentration of $250 \mu \mathrm{g} / \mathrm{mL}$. Amphicillin was served as standard. Results of inhibition zone and minimal inhibitory concentration (MIC) were noted and illustrated in Table 1.

It has been found from the results of antibacterial screening that out of all the tested compounds, three compounds 10, 11

Table 1. Antibacterial activity ${ }^{a}$ of the compounds 3-17 against tested bacterial strains

\begin{tabular}{|c|c|c|c|c|c|c|c|}
\hline Compound No. & $\mathrm{R}$ & $\begin{array}{c}\text { S. aureus } \\
\text { ATCC } 25923\end{array}$ & $\begin{array}{l}\text { B. subtilis } \\
\text { ATCC } 1633\end{array}$ & $\begin{array}{l}\text { S. epidermis } \\
\text { ATCC } 14940\end{array}$ & $\begin{array}{c}\text { E. coli } \\
\text { ATCC } 25922\end{array}$ & $\begin{array}{c}\text { K. pneumoniae } \\
\text { АТCC } 10031\end{array}$ & $\begin{array}{l}P . \text { aeruginosa } \\
\text { ATCC } 27853\end{array}$ \\
\hline 3 & & $\begin{array}{c}10 \\
(>100)\end{array}$ & $\begin{array}{c}11 \\
(>100)\end{array}$ & $\begin{array}{c}07 \\
(>100)\end{array}$ & $\begin{array}{c}09 \\
(>100)\end{array}$ & - & $\begin{array}{c}06 \\
(>100)\end{array}$ \\
\hline 4 & & $\begin{array}{c}12 \\
(>100)\end{array}$ & $\begin{array}{c}15 \\
(>100)\end{array}$ & - & $\begin{array}{c}08 \\
(>100)\end{array}$ & $\begin{array}{c}13 \\
(>100)\end{array}$ & $\begin{array}{c}17 \\
(50)\end{array}$ \\
\hline 5 & & $\begin{array}{c}16 \\
(50)\end{array}$ & $\begin{array}{c}18 \\
(>100)\end{array}$ & $\begin{array}{c}14 \\
(50)\end{array}$ & $\begin{array}{c}12 \\
(>100)\end{array}$ & $\begin{array}{c}10 \\
(>100)\end{array}$ & $\begin{array}{c}21 \\
(25)\end{array}$ \\
\hline 6 & & $\begin{array}{c}19 \\
(25)\end{array}$ & $\begin{array}{c}22 \\
(50)\end{array}$ & $\begin{array}{c}20 \\
(12.5)\end{array}$ & $\begin{array}{c}15 \\
(>100)\end{array}$ & $\begin{array}{c}18 \\
(3.125)\end{array}$ & $\begin{array}{c}23 \\
(25)\end{array}$ \\
\hline 7 & & $\begin{array}{c}09 \\
(>100)\end{array}$ & $\begin{array}{c}06 \\
(125)\end{array}$ & $\begin{array}{c}10 \\
(>100)\end{array}$ & - & $\begin{array}{c}07 \\
(>100)\end{array}$ & $\begin{array}{c}08 \\
(>100)\end{array}$ \\
\hline 8 & & $\begin{array}{l}22 \\
(25)\end{array}$ & $\begin{array}{l}25 \\
(50)\end{array}$ & $\begin{array}{c}18 \\
(25)\end{array}$ & $\begin{array}{c}17 \\
(50)\end{array}$ & $\begin{array}{c}14 \\
(25)\end{array}$ & $\begin{array}{c}25 \\
(25)\end{array}$ \\
\hline 9 & & $\begin{array}{c}26 \\
(12.5)\end{array}$ & $\begin{array}{l}29 \\
(25)\end{array}$ & $\begin{array}{c}24 \\
(6.25)\end{array}$ & $\begin{array}{c}27 \\
(12.5)\end{array}$ & $\begin{array}{c}19 \\
(3.125)\end{array}$ & $\begin{array}{c}31 \\
(6.25)\end{array}$ \\
\hline 10 & & - & $\begin{array}{c}34 \\
(6.25)\end{array}$ & $\begin{array}{c}27 \\
(6.25)\end{array}$ & $\begin{array}{c}24 \\
(25)\end{array}$ & $\begin{array}{c}27 \\
(0.781)\end{array}$ & $\begin{array}{c}30 \\
(6.25)\end{array}$ \\
\hline 11 & & $\begin{array}{c}35 \\
(1.562)\end{array}$ & $\begin{array}{c}38 \\
(3.125)\end{array}$ & $\begin{array}{c}30 \\
(3.125)\end{array}$ & $\begin{array}{c}32 \\
(6.25)\end{array}$ & $\begin{array}{c}21 \\
(3.125)\end{array}$ & $\begin{array}{c}39 \\
(1.562)\end{array}$ \\
\hline 12 & & $\begin{array}{c}21 \\
(25)\end{array}$ & $\begin{array}{c}27 \\
(25)\end{array}$ & $\begin{array}{c}23 \\
(12.5)\end{array}$ & $\begin{array}{c}20 \\
(50)\end{array}$ & - & $\begin{array}{c}28 \\
(12.5)\end{array}$ \\
\hline 13 & & $\begin{array}{c}18 \\
(25)\end{array}$ & $\begin{array}{c}21 \\
(50)\end{array}$ & - & - & $\begin{array}{c}16 \\
(6.25)\end{array}$ & $\begin{array}{c}22 \\
(25)\end{array}$ \\
\hline 14 & & $\begin{array}{c}24 \\
(12.5)\end{array}$ & $\begin{array}{c}23 \\
(50)\end{array}$ & $\begin{array}{c}21 \\
(12.5)\end{array}$ & $\begin{array}{c}22 \\
(25)\end{array}$ & - & $\begin{array}{c}27 \\
(12.5)\end{array}$ \\
\hline 15 & & $\begin{array}{c}30 \\
(6.25)\end{array}$ & $\begin{array}{c}31 \\
(12.5)\end{array}$ & $\begin{array}{c}25 \\
(6.25)\end{array}$ & $\begin{array}{c}24 \\
(25)\end{array}$ & $\begin{array}{c}20 \\
(3.125)\end{array}$ & $\begin{array}{c}30 \\
(6.25)\end{array}$ \\
\hline 16 & & $\begin{array}{c}32 \\
(3.125)\end{array}$ & $\begin{array}{c}34 \\
(6.25)\end{array}$ & $\begin{array}{c}23 \\
(12.5)\end{array}$ & $\begin{array}{c}30 \\
(6.25)\end{array}$ & $\begin{array}{c}21 \\
(3.125)\end{array}$ & $\begin{array}{c}31 \\
(6.25)\end{array}$ \\
\hline 17 & & $\begin{array}{c}17 \\
(25)\end{array}$ & $\begin{array}{c}27 \\
(25)\end{array}$ & $\begin{array}{c}21 \\
(12.5)\end{array}$ & $\begin{array}{c}19 \\
(50)\end{array}$ & $\begin{array}{c}17 \\
(6.25)\end{array}$ & $\begin{array}{c}23 \\
(25)\end{array}$ \\
\hline $\mathrm{DMSO}^{b}$ & & 0 & 0 & 0 & 0 & 0 & 0 \\
\hline Amphicillin & & $\begin{array}{c}29 \\
(6.25)\end{array}$ & $\begin{array}{c}32 \\
(12.5)\end{array}$ & $\begin{array}{c}26 \\
(6.25)\end{array}$ & $\begin{array}{c}28 \\
(12.5)\end{array}$ & $\begin{array}{c}24 \\
(1.562)\end{array}$ & $\begin{array}{c}35 \\
(3.125)\end{array}$ \\
\hline
\end{tabular}

${ }^{a}$ Concentration was $250 \mu \mathrm{g} / \mathrm{mL} .{ }^{b}$ Served as control, denotes no inhibition zone was observed. Values in brackets are MIC. 
and 16 exhibited prominent antibacterial activity against different bacterial strains (Table 1). Among these three compounds, compound $\mathbf{1 0}$ has shown substantial activity than standard drug against the bacterial strains: $B$. subtilis and $K$. pneumoniae with MIC $6.25 \mu \mathrm{g} / \mathrm{mL}$ and $0.781 \mu \mathrm{g} / \mathrm{mL}$, respectively. Compound 11 (azetidinone moiety bearing $p$-methoxyphenyl substituent) reflected excellent antibacterial activity against all the bacterial strains except $K$. pneumoniae with MIC $1.562-6.25 \mu \mathrm{g} / \mathrm{mL}$ (Table 1). Moreover, compound 16 (thiazolidinone moiety having $p$-methoxyphenyl group) showed better efficacy as compared to standard drug against $S$. aureus (MIC $3.125 \mu \mathrm{g} / \mathrm{mL}$ ), B. subtilis (MIC $6.25 \mu \mathrm{g} / \mathrm{mL}$ ) and E. coli (MIC $6.25 \mu \mathrm{g} / \mathrm{mL}$ ). Results has also indicated that compounds $\mathbf{9}$ and $\mathbf{1 5}$ displayed equipotent antibacterial activity as compared to the reference drug against all the bacterial strains tested (Table 1). The rest compounds of this series were found to be less active as compared to the standard drug.

It is significant note form the antibacterial results that the conversion of compounds 3-7 into azetidinone congeners 8-12 and thiazolidinone congeners 13-17 increased the inhibition action against the growth of various pathogens. However, compounds 8-12 consisting $\beta$-lactam ring displayed improved antibacterial activity as compared to thiazolidinone derivatives 13-17.

Furthermore, the effect of different substituents on antibacterial activity was examined and after reviewing the antibacterial results of compounds 3-17 few observations could be drawn like:

It has been observed that compounds $\mathbf{6 , 1 1}$ and 16 bearing $p$ methoxyphenyl group furnished most potent antibacterial activity in their respective class of compounds 3-7, 8-12 and 13-17, respectively.

It is interesting to point out that compounds having $o$-hydroxyphenyl group as seen in compounds 5, 10 and 15 has elicited remarkable inhibitory action.

Compounds having furfuryl $(7,12,17)$ and $o$-chlorophenyl $(4,9,14)$ groups has yielded less but still adequate antibacterial activity.

\section{Experimental}

Chemistry. All the reagents and solvents were generally received form commercial supplier. Reactions were done in dried glassware. Melting points were taken in open capillaries by thermonic melting point apparatus, (Campbell Electronic Mumbai, India) and are uncorrected. The purity of the newly synthesized compounds was checked by thin layer chromatography (TLC) on silica gel-G coated plates by using different solvent systems. Infrared (IR) spectra were determined on Bruker IFS66 FTIR (Bruker Bioscience, USA) using $\mathrm{KBr}$ pallets and wave number $(v)$ was reported in $\mathrm{cm}^{-1}$. The ${ }^{1} \mathrm{H}-\mathrm{NMR}$ spectra were taken on Jeol GSX -300 FT NMR (Jeol, Tokyo, Japan) in $\mathrm{CDCl}_{3}$ or DMSO- $d_{6}$ ' and chemical shifts $(\delta)$ are given in ppm. Tetramethylsilane (TMS) was used as internal reference standard. Mass spectra were recorded on Spec Finnigan Mat 8230 MS. The carbon, hydrogen and nitrogen analysis were performed on Carlo Erba-1108 (Carlo Erba, Milan, Italy), and the results were found with in $\pm 0.4 \%$ of the theoretical values.

General procedure for the synthesis of 8-methoxy-4-methyl- 2-chloroquinoline 1. A mixture of 8-methoxy-4-methyl-quinolin-2-ol (38.0 g; $0.75 \mathrm{~mol})$ and freshly distilled phosphorous oxychloride $(21.55 \mathrm{~mL} ; 0.9 \mathrm{~mol})$ was maintained at $80-85^{\circ} \mathrm{C}$ on water bath for 15 minutes until most of the solids have dissolved. This reaction mixture was heated for another $15 \mathrm{mi}-$ nutes. Then, this hot mixture was poured onto crushed ice containing $1 \mathrm{~L}$ of water. Further, the obtained product was extracted by using $700 \mathrm{~mL}$ ether and $200 \mathrm{~mL}$ water and then dried over $50 \mathrm{~g}$ of potassium carbonate. After the removal of the ether, the residual oil is distilled off. Then, colorless distillate boils at $132-135^{\circ} \mathrm{C}$. The distillate is melted, if necessary, and poured into $250 \mathrm{~mL}$ of petroleum ether $\left(40-45^{\circ} \mathrm{C}\right)$. The solution is then chilled in a freezing mixture; the crystals are filtered by suction and dried in a vacuum desiccator over paraffin. Compound 1: $\mathrm{mp} 65^{\circ} \mathrm{C}$; yield: $32.30 \mathrm{~g}(85.0 \%)$; IR $(\mathrm{KBr}) v$ in $\mathrm{cm}^{-1}: 3070$ (C-H aromatic ring), 2950 (C-H aliphatic), 1560 (C-----C of aromatic ring), 1160 (C-N), 1075 (C-O-C), 735 (C-Cl). ${ }^{\mathrm{T}} \mathrm{H}-\mathrm{NMR}$ $\left(\mathrm{CDCl}_{3}\right) \delta 7.92\left(\mathrm{~s}, 1 \mathrm{H}, H_{3}\right.$ of quinoline), $7.81\left(\mathrm{~d}, 1 \mathrm{H}, H_{5}\right.$ of quinoline, $J=6.0 \mathrm{~Hz}), 7.78\left(\mathrm{~d}, 1 \mathrm{H}, H_{7}\right.$ of quinoline, $\left.J=9.0 \mathrm{~Hz}\right), 7.65$ (t, $1 \mathrm{H}, H_{6}$ of quinoline, $\left.J=7.5 \mathrm{~Hz}\right), 3.49\left(\mathrm{~s}, 3 \mathrm{H}, \mathrm{OCH}_{3}\right), 2.84(\mathrm{~s}$, $3 \mathrm{H}, \mathrm{CH}_{3}$ ). MS: [M] $]^{+}$at $\mathrm{m} / \mathrm{z} 207.5$ and $[\mathrm{M}+2]$ at $\mathrm{m} / \mathrm{z} 209.5$. Anal. calcd for $\mathrm{C}_{11} \mathrm{H}_{10} \mathrm{NOCl}$ : C, 63.61; H, 4.82; N, 6.75; Found: $\mathrm{C}$, 63.79; H, 4.65; N, 6.92 .

General procedure for the synthesis of 8-methoxy-4-methyl2-hyrazinoquinoline 2. An aqueous solution of hydrazine hydrate $(99-100 \% ; 14.52 \mathrm{~mL} ; 0.02 \mathrm{~mol})$ has added to a suspension of compound 1 (31.0 g; $0.01 \mathrm{~mol})$ in ethanol $(95 \mathrm{~mL})$. The reaction mixture was refluxed for $6 \mathrm{~h}$ and then cooled at room temperature. The separated crystalline product was filtered, washed with little ethanol, dried and recrystallized form absolute ethanol to give compound 2: $\mathrm{mp} 262^{\circ} \mathrm{C}$; yield: $24.8 \mathrm{~g}(80-0 \%)$; IR $(\mathrm{KBr}) v$ in $\mathrm{cm}^{-1}: 3245(\mathrm{NH}), 3065$ (C-H aromatic ring), 2965 (C-H aliphatic), 1564 (C-----C of aromatic ring), $1174(\mathrm{C}-\mathrm{N})$, 1091 (C-O-C), 1039 (N-N). ${ }^{\mathrm{H}} \mathrm{H}-\mathrm{NMR}\left(\mathrm{CDCl}_{3}\right) \delta 9.65$ (bs, $1 \mathrm{H}$, $\mathrm{NHNH}_{2}$, exchangeable with $\left.\mathrm{D}_{2} \mathrm{O}\right), 7.93\left(\mathrm{~s}, 1 \mathrm{H}, H_{3}\right.$ of quinoline), $7.80\left(\mathrm{~d}, 1 \mathrm{H}, H_{5}\right.$ of quinoline, $\left.J=6.0 \mathrm{~Hz}\right), 7.79\left(\mathrm{~d}, 1 \mathrm{H}, H_{7}\right.$ of quinoline, $J=9.0 \mathrm{~Hz}$ ), $7.63\left(\mathrm{t}, 1 \mathrm{H}, H_{6}\right.$ of quinoline, $J=7.5 \mathrm{~Hz}$ ), $3.51\left(\mathrm{~s}, 3 \mathrm{H}, \mathrm{OCH}_{3}\right), 2.86\left(\mathrm{~s}, 3 \mathrm{H}, \mathrm{CH}_{3}\right), 4.58\left(\mathrm{~s}, 2 \mathrm{H}, \mathrm{NHNH}_{2}\right.$, exchangeable with $\mathrm{D}_{2} \mathrm{O}$ ). MS: $[\mathrm{M}]^{+}$at $m / z$ 203. Anal. calcd for $\mathrm{C}_{11} \mathrm{H}_{13} \mathrm{~N}_{3} \mathrm{O}: \mathrm{C}, 65.02 ; \mathrm{H}, 6.40 ; \mathrm{N}, 20.69$; Found: C, 64.88; H, 6.32; N, 20.51 .

General procedure for the synthesis of 8-methoxy-4-methyl2-(substituted arylidenyliminoamino)quinolines 3-7. To a solution of compound $2(0.01 \mathrm{~mol})$ in methanol $(80 \mathrm{~mL})$, aromatic aldehyde $(0.01 \mathrm{~mol})$ along with few drops of glacial acetic acid was added. This resulting mixture was refluxed for $10-12 \mathrm{~h}$, while TLC monitored progress and completion of the reaction. The volatiles were evaporated, and the remaining mixture was filtered. The solid separated out was recrystallized from appropriate solvent to get compounds 3-7.

8-Methoxy-4-methyl-2-(arylidenyl-iminoamino)quinoline 3: Reagents: compound 2 ( $4.5 \mathrm{~g}, 0.01 \mathrm{~mol})$, benzaldehyde $(2.25$ $\mathrm{mL}, 0.01 \mathrm{~mol})$, methanol $(80 \mathrm{~mL}), \mathrm{mp} 168{ }^{\circ} \mathrm{C}$; yield: $3.69 \mathrm{~g}$ (82.0\%); Recrytallization solvent: Acetone; IR $(\mathrm{KBr}) v$ in $\mathrm{cm}^{-1}$ : 3285 (NH), 3075 (C-H aromatic ring), 2964 (C-H aliphatic), 1579 (C-----C of aromatic ring), $1188(\mathrm{C}-\mathrm{N}), 1045(\mathrm{~N}-\mathrm{N}) .{ }^{1} \mathrm{H}-$ $\operatorname{NMR}\left(\overline{\mathrm{CDCl}}_{3}\right) \delta 8.11(\mathrm{~d}, 1 \mathrm{H}, \mathrm{CH}-\mathrm{A} r, J=11.0 \mathrm{~Hz}), 7.91(\mathrm{~s}, 1 \mathrm{H}$, $H_{3}$ of quinoline), 7.79 (d, $1 \mathrm{H}, H_{5}$ of quinoline, $\left.J=6.0 \mathrm{~Hz}\right), 7.74$ 
$\left(\mathrm{d}, 1 \mathrm{H}, H_{7}\right.$ of quinoline, $\left.J=9.0 \mathrm{~Hz}\right), 7.61\left(\mathrm{t}, 1 \mathrm{H}, H_{6}\right.$ of quinoline, $J=7.5 \mathrm{~Hz}), 7.25(\mathrm{~m}, 5 \mathrm{H}, \mathrm{Ar}-H), 6.65(\mathrm{~s}, 1 \mathrm{H}, \mathrm{NH}$, exchangeable with $\left.\mathrm{D}_{2} \mathrm{O}\right), 3.52\left(\mathrm{~s}, 3 \mathrm{H}, \mathrm{OCH}_{3}\right), 2.82\left(\mathrm{~s}, 3 \mathrm{H}, \mathrm{CH}_{3}\right)$. MS: [M] at $m / z$ 291. Anal. calcd for $\mathrm{C}_{18} \mathrm{H}_{17} \mathrm{~N}_{3} \mathrm{O}: \mathrm{C}, 74.23 ; \mathrm{H}, 5.84 ; \mathrm{N}$, 14.43; Found: C, 74.37; H, 5.62; N, 14.63.

8-Methoxy-4-methyl-2-(o-chloroarylidenyl-iminoamino)quinoline 4: Reagents: compound 2 (4.5 g, $0.01 \mathrm{~mol}$ ), $o$-chlorobenzaldehyde $(2.49 \mathrm{~mL}, 0.01 \mathrm{~mol})$, methanol $(80 \mathrm{~mL}), \mathrm{mp} 180{ }^{\circ} \mathrm{C}$; yield: 3.83 g (85.0\%); Recrytallization solvent: Ethanol; IR $(\mathrm{KBr}) v$ in $\mathrm{cm}^{-1}: 3256(\mathrm{NH}), 3064(\mathrm{C}-\mathrm{H}$ aromatic ring), 2962 (C-H aliphatic), 1594 (C-----C of aromatic ring), $1187(\mathrm{C}-\mathrm{N})$, $1074(\mathrm{~N}-\mathrm{N}), 765(\mathrm{C}-\mathrm{Cl}) .{ }^{1} \mathrm{H}-\mathrm{NMR}$ (DMSO-d $\left.d_{6}\right) \delta 8.13(\mathrm{~d}, 1 \mathrm{H}$, $\mathrm{CH}-\mathrm{Ar}, J=11.0 \mathrm{~Hz}), 7.89$ (s, $1 \mathrm{H}, H_{3}$ of quinoline), $7.81(\mathrm{~d}, 1 \mathrm{H}$, $H_{5}$ of quinoline, $\left.J=6.0 \mathrm{~Hz}\right), 7.72\left(\mathrm{~d}, 1 \mathrm{H}, H_{7}\right.$ of quinoline, $J=$ $9.0 \mathrm{~Hz}), 7.63\left(\mathrm{t}, 1 \mathrm{H}, H_{6}\right.$ of quinoline, $\left.J=7.5 \mathrm{~Hz}\right), 7.22-7.45$ $(\mathrm{m}, 4 \mathrm{H}, \mathrm{Ar}-H), 6.65\left(\mathrm{~s}, 1 \mathrm{H}, \mathrm{N} H\right.$, exchangeable with $\left.\mathrm{D}_{2} \mathrm{O}\right), 3.51$ (s, 3H, $\left.\mathrm{OCH}_{3}\right), 2.81\left(\mathrm{~s}, 3 \mathrm{H}, \mathrm{CH}_{3}\right)$. MS: $[\mathrm{M}]^{+}$at $\mathrm{m} / z 325.5$ and $[\mathrm{M}+2]$ at $m / z$ 327.5. Anal. calcd for $\mathrm{C}_{18} \mathrm{H}_{16} \mathrm{~N}_{3} \mathrm{OCl}: \mathrm{C}, 66.36$; H, 4.92; N, 12.90; Found: C, 66.50; H, 4.76; N, 13.12.

8-Methoxy-4-methyl-2-(o-hydroxyarylidenyl-iminoamino) quinoline 5: Reagents: compound 2 (4.5 g, $0.01 \mathrm{~mol}), o$-hydroxybenzaldehyde $(2.36 \mathrm{~mL}, 0.01 \mathrm{~mol})$, methanol $(80 \mathrm{~mL}), \mathrm{mp}$ $225{ }^{\circ} \mathrm{C}$; yield: $3.38 \mathrm{~g} \mathrm{(75.0 \% )}$; Recrytallization solvent: Ethyl acetate; IR (KBr) $v$ in $\mathrm{cm}^{-1}$ : $3565(\mathrm{OH}), 3274(\mathrm{NH}), 3075(\mathrm{C}-\mathrm{H}$ aromatic ring), 2961 (C-H aliphatic), 1582 (C-----C of aromatic ring), $1191(\mathrm{C}-\mathrm{N}), 1065(\mathrm{~N}-\mathrm{N}) .{ }^{1} \mathrm{H}-\mathrm{NMR}\left(\mathrm{CDCl}_{3}\right) \delta 10.11(\mathrm{~s}$, $1 \mathrm{H}, \mathrm{OH}$, exchangeable with $\left.\mathrm{D}_{2} \mathrm{O}\right), 8.14(\mathrm{~d}, 1 \mathrm{H}, \mathrm{CH}-\mathrm{A} r, J=$ $11.0 \mathrm{~Hz}), 7.92\left(\mathrm{~s}, 1 \mathrm{H}, H_{3}\right.$ of quinoline $), 7.81\left(\mathrm{~d}, 1 \mathrm{H}, H_{5}\right.$ of quinoline, $J=6.0 \mathrm{~Hz}), 7.78\left(\mathrm{~d}, 1 \mathrm{H}, H_{7}\right.$ of quinoline, $\left.J=9.0 \mathrm{~Hz}\right)$, $7.64\left(\mathrm{t}, 1 \mathrm{H}, H_{6}\right.$ of quinoline, $\left.J=7.5 \mathrm{~Hz}\right), 7.2-7.46(\mathrm{~m}, 4 \mathrm{H}, \mathrm{Ar}-H)$, $6.63\left(\mathrm{~s}, 1 \mathrm{H}, \mathrm{N} H\right.$, exchangeable with $\left.\mathrm{D}_{2} \mathrm{O}\right), 3.52\left(\mathrm{~s}, 3 \mathrm{H}, \mathrm{OCH}_{3}\right)$, 2.83 (s, 3H, $\mathrm{CH}_{3}$ ). MS: [M] $]^{+}$at $m / z$ 307. Anal. calcd for $\mathrm{C}_{18} \mathrm{H}_{17^{-}}$ $\mathrm{N}_{3} \mathrm{O}_{2}$ : C, 70.36; H, 5.54; N, 13.68; Found: C, 70.46; H, 5.30; N, 13.79 .

8-Methoxy-4-methyl-2-(o-methoxyarylidenyl-iminoamino) quinoline 6: Reagents: compound 2 (4.5 g, $0.01 \mathrm{~mol})$, 2-methoxybenzaldehyde $(2.70 \mathrm{~mL}, 0.01 \mathrm{~mol})$, methanol $(80 \mathrm{~mL}), \mathrm{mp}$ $210{ }^{\circ} \mathrm{C}$; yield: $3.87 \mathrm{~g}(86.0 \%)$; Recrytallization solvent : Methanol; IR (KBr) $v$ in $\mathrm{cm}^{-1}: 3265(\mathrm{NH}), 3055$ (C-H aromatic ring), 2974 (C-H aliphatic), 1572 (C-----C of aromatic ring), 1177 $(\mathrm{C}-\mathrm{N}), 1071$ (C-O-C), $1045(\mathrm{~N}-\overline{\mathrm{N}}) .{ }^{\mathrm{T}} \mathrm{H}-\mathrm{NMR}$ (DMSO-d $\left.{ }_{6}\right) \delta 8.12$ (d, $1 \mathrm{H}, \mathrm{CH}-\mathrm{Ar}, J=11.0 \mathrm{~Hz}$ ), 7.90 (s, $1 \mathrm{H}, H_{3}$ of quinoline), 7.80 $\left(\mathrm{d}, 1 \mathrm{H}, H_{5}\right.$ of quinoline, $\left.J=6.0 \mathrm{~Hz}\right), 7.79\left(\mathrm{~d}, 1 \mathrm{H}, H_{7}\right.$ of quinoline, $J=9.0 \mathrm{~Hz}), 7.63\left(\mathrm{t}, 1 \mathrm{H}, H_{6}\right.$ of quinoline, $\left.J=7.5 \mathrm{~Hz}\right), 7.22-$ $7.45(\mathrm{~m}, 4 \mathrm{H}, \mathrm{Ar}-H), 6.65$ (s, $1 \mathrm{H}, \mathrm{N} H$, exchangeable with $\left.\mathrm{D}_{2} \mathrm{O}\right)$, $3.51\left(\mathrm{~s}, 3 \mathrm{H}, \mathrm{OCH}_{3}\right), 3.21\left(\mathrm{~s}, 3 \mathrm{H}, \mathrm{OCH}_{3}\right), 2.81\left(\mathrm{~s}, 3 \mathrm{H}, \mathrm{CH}_{3}\right)$. MS: $[\mathrm{M}]^{+}$at $m / z$ 321. Anal. calcd for $\mathrm{C}_{19} \mathrm{H}_{19} \mathrm{~N}_{3} \mathrm{O}_{2}$ : C, 71.03; H, 5.92; N, 13.04; Found: C, 70.95; H, 5.84; N, 13.15 .

8-Methoxy-4-methyl-2-(furfurylidenyl-iminoamino)quinoline 7: Reagents: compound 2 ( $4.5 \mathrm{~g}, 0.01 \mathrm{~mol})$, furfuraldehyde $(1.84 \mathrm{~mL}, 0.01 \mathrm{~mol})$, methanol $(80 \mathrm{~mL}) ; \mathrm{mp} 191{ }^{\circ} \mathrm{C}$; yield: $3.33 \mathrm{~g} \mathrm{(74.0 \% );} \mathrm{Recrytallization} \mathrm{solvent:} \mathrm{Acetic} \mathrm{acid;} \mathrm{IR} \mathrm{(KBr)}$ $v$ in $\mathrm{cm}^{-1}: 3288(\mathrm{NH}), 3045$ (C-H aromatic ring), $2964(\mathrm{C}-\mathrm{H}$ aliphatic), 1552 (C-----C of aromatic ring), 1187 (C-N), 1081 (C-O-C), $1064(\mathrm{~N}-\mathrm{N}) .{ }^{\mathrm{T}} \mathrm{H}-\mathrm{NMR}$ (DMSO-d $) \delta 8.13(\mathrm{~d}, 1 \mathrm{H}, \mathrm{CH}-$ $\mathrm{A} r, J=11.0 \mathrm{~Hz}), 7.91\left(\mathrm{~s}, 1 \mathrm{H}, H_{3}\right.$ of quinoline), $7.82\left(\mathrm{~d}, 1 \mathrm{H}, H_{5}\right.$ of quinoline, $J=6.0 \mathrm{~Hz}), 7.78\left(\mathrm{~d}, 1 \mathrm{H}, H_{7}\right.$ of quinoline, $\left.J=9.0 \mathrm{~Hz}\right)$, $7.65\left(\mathrm{t}, 1 \mathrm{H}, H_{6}\right.$ of quinoline, $\left.J=7.5 \mathrm{~Hz}\right), 7.13-7.32(\mathrm{~m}, 3 \mathrm{H}$,
Ar- $H), 6.67$ (s, $1 \mathrm{H}, \mathrm{N} H$, exchangeable with $\left.\mathrm{D}_{2} \mathrm{O}\right), 3.51(\mathrm{~s}, 3 \mathrm{H}$, $\left.\mathrm{OCH}_{3}\right), 2.81\left(\mathrm{~s}, 3 \mathrm{H}, \mathrm{CH}_{3}\right)$. MS: [M] ${ }^{+}$at $m / z 281$. Anal. calcd for $\mathrm{C}_{16} \mathrm{H}_{15} \mathrm{~N}_{3} \mathrm{O}_{2}$ : C, 68.32; H, 5.33; N, 14.94; Found: C, 68.41; H, $5.26 ; \mathrm{N}, 14.87$.

General procedure for the synthesis of 8-methoxy-4-methyl2-amino-(3'-chloro-2'-oxo-4'-substituted aryl-1'-azetidinyl)quinolines 8-12: To a solution of compound (3-7, $0.01 \mathrm{~mol})$ in DMF $(60 \mathrm{~mL})$, chloroacetyl chloride $(0.02 \mathrm{~mol})$ and triethyl amine $(0.02 \mathrm{~mol})$ were added at $0-5{ }^{\circ} \mathrm{C}$ temperature with constant stirring. This reaction mixture was refluxed on water bath for $6-8 \mathrm{~h}$; then excess of solvent was distilled off. The precipitated product was cooled, poured in ice-water then filtered, further recrystallized from appropriate solvent to procure compounds 8-12.

8-Methoxy-4-methyl-2-amino-[3'-chloro-2'-oxo-4'-(phenyl)1'-azetidinyl]quinoline 8: Reagents: compound 3 (1.6 g, 0.01 $\mathrm{mol})$, chloroacetyl chloride $(0.87 \mathrm{~mL}, 0.02 \mathrm{~mol})$, triethyl amine (1.53 mL, $0.02 \mathrm{~mol}), \mathrm{DMF}(60 \mathrm{~mL}) ; \mathrm{mp} 112^{\circ} \mathrm{C}$; yield: $0.96 \mathrm{~g}$ (60.0\%); Recrytallization solvent: Ethanol; IR (KBr) $v$ in $\mathrm{cm}^{-1}$ : 3261 (NH), 3055 (C-H aromatic ring), 2981 (C-H aliphatic), $1765(\mathrm{C}=\mathrm{O}), 1575$ (C-----C of aromatic ring), $1155(\mathrm{C}-\mathrm{N}), 1064$ $(\mathrm{N}-\mathrm{N}), 761(\mathrm{C}-\mathrm{Cl}) .{ }^{1} \mathrm{H}-\mathrm{N} M R\left(\mathrm{DMSO}-d_{6}\right) \delta 8.12(\mathrm{~d}, 1 \mathrm{H}, \mathrm{CH}-$ $\mathrm{A} r, J=11.0 \mathrm{~Hz}), 7.90\left(\mathrm{~s}, 1 \mathrm{H}, H_{3}\right.$ of quinoline), $7.81\left(\mathrm{~d}, 1 \mathrm{H}, H_{5}\right.$ of quinoline, $J=6.0 \mathrm{~Hz}), 7.79\left(\mathrm{~d}, 1 \mathrm{H}, H_{7}\right.$ of quinoline, $J=9.0$ $\mathrm{Hz}), 7.62\left(\mathrm{t}, 1 \mathrm{H}, H_{6}\right.$ of quinoline, $\left.J=7.5 \mathrm{~Hz}\right), 7.27(\mathrm{~m}, 5 \mathrm{H}$, Ar- $H), 6.63\left(\mathrm{~s}, 1 \mathrm{H}, \mathrm{N} H\right.$, exchangeable with $\left.\mathrm{D}_{2} \mathrm{O}\right), 6.52(\mathrm{~d}, 1 \mathrm{H}$, $\mathrm{CH}-\mathrm{Cl} J=6.0 \mathrm{~Hz}), 3.54\left(\mathrm{~s}, 3 \mathrm{H}, \mathrm{OCH}_{3}\right), 2.81\left(\mathrm{~s}, 3 \mathrm{H}, \mathrm{CH}_{3}\right)$. MS: $[\mathrm{M}]^{+}$at $\mathrm{m} / z 367.5$ and $[\mathrm{M}+2]$ at $\mathrm{m} / z$ 369.5. Anal. calcd for $\mathrm{C}_{20} \mathrm{H}_{18} \mathrm{~N}_{3} \mathrm{O}_{2} \mathrm{Cl}: \mathrm{C}, 65.31 ; \mathrm{H}, 4.90 ; \mathrm{N}, 11.43$; Found: C, 65.50; $\mathrm{H}, 4.72 ; \mathrm{N}, 11.55$.

8-Methoxy-4-methyl-2-amino-[3'-chloro-2'-oxo-4'-(o-chlrophenyl)-1'-azetidinyl]quinoline 9: eagents: compound 4 (1.7 g, $0.01 \mathrm{~mol})$, chloroacetyl chloride $(0.83 \mathrm{~mL}, 0.02 \mathrm{~mol})$, triethyl amine (1.46 mL, $0.02 \mathrm{~mol}), \mathrm{DMF}(60 \mathrm{~mL}) ; \mathrm{mp} 171{ }^{\circ} \mathrm{C}$; yield: $1.11 \mathrm{~g}(65.0 \%)$; Recrytallization solvent: Acetic acid; IR (KBr) $v$ in $\mathrm{cm}^{-1}: 3254(\mathrm{NH}), 3044$ (C-H aromatic ring), 2947 (C-H aliphatic), $1776(\mathrm{C}=\mathrm{O}), 1585$ (C-----C of aromatic ring), 1154 $(\mathrm{C}-\mathrm{N}), 1068(\mathrm{~N}-\mathrm{N}), 785(\mathrm{C}-\mathrm{Cl}) .{ }^{\mathrm{H}-\mathrm{N} M R}\left(\mathrm{CDCl}_{3}\right) \delta 8.13(\mathrm{~d}, 1 \mathrm{H}$, $\mathrm{CH}-\mathrm{Ar}, J=11.0 \mathrm{~Hz}), 7.89\left(\mathrm{~s}, 1 \mathrm{H}, H_{3}\right.$ of quinoline), $7.80(\mathrm{~d}, 1 \mathrm{H}$, $H_{5}$ of quinoline, $\left.J=6.0 \mathrm{~Hz}\right), 7.77\left(\mathrm{~d}, 1 \mathrm{H}, H_{7}\right.$ of quinoline, $J=$ $9.0 \mathrm{~Hz}), 7.61\left(\mathrm{t}, 1 \mathrm{H}, H_{6}\right.$ of quinoline, $\left.J=7.5 \mathrm{~Hz}\right), 7.22-7.41$ $(\mathrm{m}, 4 \mathrm{H}, \mathrm{Ar}-H), 6.61\left(\mathrm{~s}, 1 \mathrm{H}, \mathrm{N} H\right.$, exchangeable with $\left.\mathrm{D}_{2} \mathrm{O}\right), 6.53$ $(\mathrm{d}, 1 \mathrm{H}, \mathrm{CH}-\mathrm{Cl} J=6.0 \mathrm{~Hz}), 3.54\left(\mathrm{~s}, 3 \mathrm{H}, \mathrm{OCH}_{3}\right), 2.78(\mathrm{~s}, 3 \mathrm{H}$, $\mathrm{CH}_{3}$ ). MS: $[\mathrm{M}]^{+}$at $m / z 402$ and $[\mathrm{M}+2]$ at $m / z$ 404. Anal. calcd for $\mathrm{C}_{20} \mathrm{H}_{17} \mathrm{~N}_{3} \mathrm{O}_{2} \mathrm{Cl}_{2}$ : C, 59.70; H, 4.23; N, 10.45; Found: C, 59.50; H, 4.33; N, 10.60 .

8-Methoxy-4-methyl-2-amino-[3'-chloro-2'-oxo-4'-(o-hydroxyphenyl)-1'-azetidinyl]quinoline 10: eagents: compound 5 (1.5 g, $0.01 \mathrm{~mol})$, chloroacetyl chloride $(0.78 \mathrm{~mL}, 0.02 \mathrm{~mol})$, triethyl amine $(1.36 \mathrm{~mL}, 0.02 \mathrm{~mol}), \mathrm{DMF}(60 \mathrm{~mL}) ; \mathrm{mp} 216^{\circ} \mathrm{C}$; yield: $1.05 \mathrm{~g}(70.0 \%)$; Recrytallization solvent: Methanol; IR $(\mathrm{KBr}) v$ in $\mathrm{cm}^{-1}: 3565(\mathrm{OH}), 3271(\mathrm{NH}), 3065(\mathrm{C}-\mathrm{H}$ aromatic ring), 2941 (C-H aliphatic), $1755(\mathrm{C}=\mathrm{O}), 1575$ (C-----C of aromatic ring), $1167(\mathrm{C}-\mathrm{N}), 1055(\mathrm{C}-\mathrm{O}-\mathrm{C}), 1031(\mathrm{~N}-\mathrm{N}), 743(\mathrm{C}-\mathrm{Cl})$. ${ }^{1} \mathrm{H}-\mathrm{NMR}$ (DMSO- $\left.d_{6}\right) \delta 10.13(\mathrm{~s}, 1 \mathrm{H}, \mathrm{OH}$, exchangeable with $\left.\mathrm{D}_{2} \mathrm{O}\right), 8.12(\mathrm{~d}, 1 \mathrm{H}, \mathrm{CH}-\mathrm{A} r, J=11.0 \mathrm{~Hz}), 7.89\left(\mathrm{~s}, 1 \mathrm{H}, H_{3}\right.$ of quinoline), $7.83\left(\mathrm{~d}, 1 \mathrm{H}, H_{5}\right.$ of quinoline, $\left.J=6.0 \mathrm{~Hz}\right), 7.76(\mathrm{~d}, 1 \mathrm{H}$, $\mathrm{H}_{7}$ of quinoline, $\left.J=9.0 \mathrm{~Hz}\right), 7.66\left(\mathrm{t}, 1 \mathrm{H}, \mathrm{H}_{6}\right.$ of quinoline, $J=$ 7.5 Hz), 7.20-7.39 (m, 4H, Ar- $H$ ), 6.61 (s, $1 \mathrm{H}, \mathrm{N} H$, exchange- 
able with $\left.\mathrm{D}_{2} \mathrm{O}\right), 6.55(\mathrm{~d}, 1 \mathrm{H}, \mathrm{CH}-\mathrm{Cl} J=6.0 \mathrm{~Hz}), 3.51(\mathrm{~s}, 3 \mathrm{H}$, $\left.\mathrm{OCH}_{3}\right), 2.77$ (s, 3H, $\mathrm{CH}_{3}$ ). MS: [M] $]^{+}$at $m / z 383.5$ and $[\mathrm{M}+2]$ at $m / z$ 385.5. Anal. calcd for $\mathrm{C}_{20} \mathrm{H}_{18} \mathrm{~N}_{3} \mathrm{O}_{3} \mathrm{Cl}: \mathrm{C}, 62.58 ; \mathrm{H}, 4.69$; N, 10.95; Found: C, 62.74; H, 4.48; N, 10.70.

8-Methoxy-4-methyl-2-amino-[3'-chloro-2'-oxo-4'-(p-methoxyphenyl)-1'-azetidinyl]quinoline 11: Reagents: compound 6 (1.7 g, $0.01 \mathrm{~mol})$, chloroacetyl chloride $(0.84 \mathrm{~mL}, 0.02 \mathrm{~mol})$, triethyl amine $(1.48 \mathrm{~mL}, 0.02 \mathrm{~mol}), \mathrm{DMF}(60 \mathrm{~mL}) ; \mathrm{mp} 193{ }^{\circ} \mathrm{C}$; yield: $1.22 \mathrm{~g} \mathrm{(66.0 \% );} \mathrm{Recrytallization} \mathrm{solvent} \mathrm{:} \mathrm{Acetone;} \mathrm{IR}$ $(\mathrm{KBr}) v$ in $\mathrm{cm}^{-1}: 3271(\mathrm{NH}), 3075(\mathrm{C}-\mathrm{H}$ aromatic ring), 2971 (C-H aliphatic), $1745(\mathrm{C}=\mathrm{O}), 1585$ (C-----C of aromatic ring), 1157 (C-N), 1085 (C-O-C), 1064 (N-N), 761 (C-Cl). ${ }^{1} \mathrm{H}-\mathrm{NMR}$ $\left(\mathrm{CDCl}_{3}\right) \delta 8.11(\mathrm{~d}, 1 \mathrm{H}, \mathrm{CH}-\mathrm{A} r, J=11.0 \mathrm{~Hz}), 7.91\left(\mathrm{~s}, 1 \mathrm{H}, H_{3}\right.$ of quinoline), $7.82\left(\mathrm{~d}, 1 \mathrm{H}, H_{5}\right.$ of quinoline, $\left.J=6.0 \mathrm{~Hz}\right), 7.78(\mathrm{~d}$, $1 \mathrm{H}, H_{7}$ of quinoline, $\left.J=9.0 \mathrm{~Hz}\right), 7.64\left(\mathrm{t}, 1 \mathrm{H}, H_{6}\right.$ of quinoline, $J=7.5 \mathrm{~Hz}$ ), 7.19-7.41 (m, 4H, Ar- $H$ ), 6.63 (s, 1H, NH, exchangeable with $\left.\mathrm{D}_{2} \mathrm{O}\right), 6.54(\mathrm{~d}, 1 \mathrm{H}, \mathrm{CH}-\mathrm{Cl} J=6.0 \mathrm{~Hz}), 3.53(\mathrm{~s}, 3 \mathrm{H}$, $\left.\mathrm{OCH}_{3}\right), 3.18\left(\mathrm{~s}, 3 \mathrm{H}, \mathrm{OCH}_{3}\right), 2.79\left(\mathrm{~s}, 3 \mathrm{H}, \mathrm{CH}_{3}\right) . \mathrm{MS}:[\mathrm{M}]^{+}$at $m / z$ 397.5 and [M+2] at $m / z$ 399.5. Anal. calcd for $\mathrm{C}_{21} \mathrm{H}_{20} \mathrm{~N}_{3} \mathrm{O}_{3} \mathrm{Cl}$ : C, 63.40; H, 5.03; N, 10.57; Found: C, 63.64; H, 4.85; N, 10.70.

8-Methoxy-4-methyl-2-amino-[3'-chloro-2'-oxo-4'-(furfuryl)-1'-azetidinyl]quinoline 12: Reagents: compound 7 (1.5 g, $0.01 \mathrm{~mol})$, chloroacetyl chloride $(0.85 \mathrm{~mL}, 0.02 \mathrm{~mol})$, triethyl amine (1.49 mL, $0.02 \mathrm{~mol}), \mathrm{DMF}(60 \mathrm{~mL}) ; \mathrm{mp} 179{ }^{\circ} \mathrm{C}$; yield: $0.92 \mathrm{~g}(61.0 \%)$; Recrytallization solvent: Methanol; IR (KBr) $v$ in $\mathrm{cm}^{-1}$ : $3284(\mathrm{NH}), 3043$ (C-H aromatic ring), 2961 (C-H aliphatic), $1785(\mathrm{C}=\mathrm{O}), 1575(\mathrm{C}-----\mathrm{C}$ of aromatic ring), 1163 (C-N), 1075 (C-O-C), $1043(\overline{\mathrm{N}-\mathrm{N}}), 758$ (C-Cl). ${ }^{1} \mathrm{H}-\mathrm{NMR}$ $\left(\mathrm{DMSO}-d_{6}\right) \delta 8.13(\mathrm{~d}, 1 \mathrm{H}, \mathrm{CH}-\mathrm{A} r, J=11.0 \mathrm{~Hz}), 7.93(\mathrm{~s}, 1 \mathrm{H}$, $H_{3}$ of quinoline), $7.81\left(\mathrm{~d}, 1 \mathrm{H}, H_{5}\right.$ of quinoline, $\left.J=9.0 \mathrm{~Hz}\right)$, $7.75\left(\mathrm{~d}, 1 \mathrm{H}, H_{7}\right.$ of quinoline, $\left.J=9.0 \mathrm{~Hz}\right), 7.66\left(\mathrm{t}, 1 \mathrm{H}, H_{6}\right.$ of quinoline, $J=7.5 \mathrm{~Hz}), 7.14-7.33(\mathrm{~m}, 3 \mathrm{H}, \mathrm{Ar}-H), 6.64(\mathrm{~s}, 1 \mathrm{H}$, $\mathrm{N} H$, exchangeable with $\left.\mathrm{D}_{2} \mathrm{O}\right), 6.52(\mathrm{~d}, 1 \mathrm{H}, \mathrm{CH}-\mathrm{Cl} J=6.0 \mathrm{~Hz})$, 3.54 (s, 3H, $\left.\mathrm{OCH}_{3}\right), 2.78\left(\mathrm{~s}, 3 \mathrm{H}, \mathrm{CH}_{3}\right)$. MS: $[\mathrm{M}]^{+}$at $m / z 357.5$ and $[\mathrm{M}+2]$ at $m / z$ 359.5. Anal. calcd for $\mathrm{C}_{18} \mathrm{H}_{16} \mathrm{~N}_{3} \mathrm{O}_{3} \mathrm{Cl}: \mathrm{C}$, 60.42; H, 4.48; N, 11.75; Found: C, 60.53; H, 4.53; N, 11.81.

General procedure for the synthesis of 8-methoxy-4-methyl2-amino-(2'-substituted aryl-4'-oxo-1',3'-thiazolidin-3'-yl)quinolines 13-17: A solution of compound (3-7, $0.01 \mathrm{~mol})$ and thioglycolic acid $(0.01 \mathrm{~mol})$ in dry dioxane $(60 \mathrm{~mL})$ in presence of anhydrous $\mathrm{ZnCl}_{2}(2-0 \mathrm{~g})$ was refluxed for 9 - 11 h. Reaction was routinely followed by TLC. After completion of the reaction, excess of solvent was removed through distillation, and solid thus obtained was poured onto crushed ice, then filtered, dried and recrystallized from appropriate solvent to yield the compounds 13-17.

8-Methoxy-4-methyl-2-amino-[2'-(phenyl)-4'-oxo-1',3'thiazolidin-3'-yl]quinoline 13: Reagents: compound 3 (1.6 g, $0.01 \mathrm{~mol})$, thioglycolic acid $(0.38 \mathrm{~mL}, 0.01 \mathrm{~mol})$, zinc chloride $(2.0 \mathrm{~g})$, dioxane $(60 \mathrm{~mL})$; mp $127^{\circ} \mathrm{C}$; yield: $1.09 \mathrm{~g}(68.0 \%)$; Recrytallization solvent: DMF; IR (KBr) $v$ in $\mathrm{cm}^{-1}: 3265(\mathrm{NH})$, 3081 (C-H aromatic ring), 2954 (C-H aliphatic), $1734(\mathrm{C}=\mathrm{O})$, 1554 (C-----C of aromatic ring), $1158(\mathrm{C}-\mathrm{N}), 1075(\mathrm{~N}-\mathrm{N}), 695$ (C-S-C). ${ }^{\mathrm{T}} \mathrm{H}-\mathrm{NMR}\left(\mathrm{CDCl}_{3}\right) \delta 8.13(\mathrm{~d}, 1 \mathrm{H}, \mathrm{CH}-\mathrm{A} r, J=11.0 \mathrm{~Hz})$, $7.91\left(\mathrm{~s}, 1 \mathrm{H}, H_{3}\right.$ of quinoline $), 7.80\left(\mathrm{~d}, 1 \mathrm{H}, H_{5}\right.$ of quinoline, $J=$ $6.0 \mathrm{~Hz}), 7.73\left(\mathrm{~d}, 1 \mathrm{H}, H_{7}\right.$ of quinoline, $\left.J=9.0 \mathrm{~Hz}\right), 7.61(\mathrm{t}, 1 \mathrm{H}$, $H_{6}$ of quinoline, $\left.J=7.5 \mathrm{~Hz}\right), 7.28(\mathrm{~m}, 5 \mathrm{H}, \operatorname{Ar}-H), 6.61(\mathrm{~s}, 1 \mathrm{H}$, $\mathrm{NH}$, exchangeable with $\left.\mathrm{D}_{2} \mathrm{O}\right), 3.73\left(\mathrm{~s}, 2 \mathrm{H}, \mathrm{CH}_{2}\right.$ of thiazoli- dinone), $3.55\left(\mathrm{~s}, 3 \mathrm{H}, \mathrm{OCH}_{3}\right), 2.76\left(\mathrm{~s}, 3 \mathrm{H}, \mathrm{CH}_{3}\right) . \mathrm{MS}$ : $[\mathrm{M}]^{+}$at $m / z$ 365. Anal. calcd for $\mathrm{C}_{20} \mathrm{H}_{19} \mathrm{~N}_{3} \mathrm{O}_{2} \mathrm{~S}: \mathrm{C}, 65.75 ; \mathrm{H}, 5.21 ; \mathrm{N}$, 11.51; Found: C, 65.88; H, 5.10; N, 11.62 .

8-Methoxy-4-methyl-2-amino-[2'-(o-chlrophenyl)-4'-oxo-1', 3'-thiazolidin-3'-yl] quinoline 14: Reagents: compound 4 (1.7 g, $0.01 \mathrm{~mol})$, thioglycolic acid $(0.36 \mathrm{~mL}, 0.01 \mathrm{~mol})$, zinc chloride (2.0 g), dioxane $(60 \mathrm{~mL})$; mp $198^{\circ} \mathrm{C}$; yield: $1.07 \mathrm{~g}(63.0 \%)$; Recrytallization solvent: Methanol; IR $(\mathrm{KBr}) v$ in $\mathrm{cm}^{-1}: 3275$ (NH), 3085 (C-H aromatic ring), 2982 (C-H aliphatic), 1752 $(\mathrm{C}=\mathrm{O}), 1534(\mathrm{C}-----\mathrm{C}$ of aromatic ring $), 1157(\mathrm{C}-\mathrm{N}), 1065$ $(\mathrm{N}-\mathrm{N}), 765(\mathrm{C}-\mathrm{Cl}), 635$ (C-S-C). ${ }^{1} \mathrm{H}-\mathrm{NMR}$ (DMSO-d 6 ) $\delta 8.12$ (d, $1 \mathrm{H}, \mathrm{CH}-\mathrm{A} r, J=11.0 \mathrm{~Hz}), 7.89$ (s, $1 \mathrm{H}, H_{3}$ of quinoline), 7.83 $\left(\mathrm{d}, 1 \mathrm{H}, H_{5}\right.$ of quinoline, $\left.J=6.0 \mathrm{~Hz}\right), 7.78\left(\mathrm{~d}, 1 \mathrm{H}, H_{7}\right.$ of quinoline, $J=9.0 \mathrm{~Hz}$ ), $7.63\left(\mathrm{t}, 1 \mathrm{H}, H_{6}\right.$ of quinoline, $J=7.5 \mathrm{~Hz}$ ), 7.20-7.38 (m, 4H, Ar- $H), 6.62$ (s, 1H, NH, exchangeable with $\left.\mathrm{D}_{2} \mathrm{O}\right), 3.72\left(\mathrm{~s}, 2 \mathrm{H}, \mathrm{CH}_{2}\right.$ of thiazolidinone), $3.53\left(\mathrm{~s}, 3 \mathrm{H}, \mathrm{OCH}_{3}\right)$, 2.73 (s, 3H, $\mathrm{CH}_{3}$ ). MS: [M] $]^{+}$at $\mathrm{m} / z 399.5$ and $[\mathrm{M}+2]$ at $\mathrm{m} / z$ 401.5. Anal. calcd for $\mathrm{C}_{20} \mathrm{H}_{18} \mathrm{~N}_{3} \mathrm{O}_{2} \mathrm{SCl}: \mathrm{C}, 60.08 ; \mathrm{H}, 4.51$; , 10.51; Found: C, 60.12; H, 4.68; N, 10.40.

8-Methoxy-4-methyl-2-amino-[2'-(o-hydroxyphenyl)-4'oxo-1',3'-thiazolidin-3'-yl]quinoline 15: Reagents: compound $5(1.5 \mathrm{~g}, 0.01 \mathrm{~mol})$, thioglycolic acid $(0.34 \mathrm{~mL}, 0.01 \mathrm{~mol})$, zinc chloride $(2.0 \mathrm{~g})$, dioxane $(60 \mathrm{~mL}) ; \mathrm{mp} 247{ }^{\circ} \mathrm{C}$; yield: $0.98 \mathrm{~g}$ (65.0\%); Recrytallization solvent: Ethanol; IR $(\mathrm{KBr}) v$ in $\mathrm{cm}^{-1}$ : $3355(\mathrm{OH}), 3261(\mathrm{NH}), 3043$ (C-H aromatic ring), 2949 (C-H aliphatic), $1735(\mathrm{C}=\mathrm{O}), 1569(\mathrm{C}-----\mathrm{C}$ of aromatic ring $), 1179$ (C-N), 1082 (C-O-C), 1045 (N-N), 671 (C-S-C). ${ }^{1} \mathrm{H}-\mathrm{NMR}$ $\left(\mathrm{CDCl}_{3}\right) \delta 10.11\left(\mathrm{~s}, 1 \mathrm{H}, \mathrm{OH}\right.$, exchangeable with $\left.\mathrm{D}_{2} \mathrm{O}\right), 8.13(\mathrm{~d}$, $1 \mathrm{H}, \mathrm{CH}-\mathrm{A} r, J=11.0 \mathrm{~Hz}$ ), $7.92\left(\mathrm{~s}, 1 \mathrm{H}, H_{3}\right.$ of quinoline), 7.83 (d, $1 \mathrm{H}, H_{5}$ of quinoline, $\left.J=6.0 \mathrm{~Hz}\right), 7.74\left(\mathrm{~d}, 1 \mathrm{H}, H_{7}\right.$ of quinoline, $J=9.0 \mathrm{~Hz}), 7.63\left(\mathrm{t}, 1 \mathrm{H}, H_{6}\right.$ of quinoline, $\left.J=7.5 \mathrm{~Hz}\right), 7.22-7.41$ (m, 4H, Ar- $H$ ), $6.64\left(\mathrm{~s}, 1 \mathrm{H}, \mathrm{N} H\right.$, exchangeable with $\left.\mathrm{D}_{2} \mathrm{O}\right), 3.73$ (s, $2 \mathrm{H}, \mathrm{CH}_{2}$ of thiazolidinone), $3.52\left(\mathrm{~s}, 3 \mathrm{H}, \mathrm{OCH}_{3}\right), 2.74(\mathrm{~s}, 3 \mathrm{H}$, $\mathrm{CH}_{3}$ ). MS: $[\mathrm{M}]^{+}$at $m / z$ 381. Anal. calcd for $\mathrm{C}_{20} \mathrm{H}_{19} \mathrm{~N}_{3} \mathrm{O}_{3} \mathrm{~S}: \mathrm{C}$, 62.99; H, 4.99; N, 11.02; Found: C, 62.85; H, 5.10; N, 11.15.

8-Methoxy-4-methyl-2-amino-[2'-(p-methoxyphenyl)-4'oxo-1',3'-thiazolidin-3'-yl]quinoline 16: Reagents: compound $\mathbf{6}(1.7 \mathrm{~g}, 0.01 \mathrm{~mol})$, thioglycolic acid $(0.37 \mathrm{~mL}, 0.01 \mathrm{~mol})$, zinc chloride $(2.0 \mathrm{~g})$, dioxane $(60 \mathrm{~mL}) ; \mathrm{mp} 207{ }^{\circ} \mathrm{C}$; yield: $1.14 \mathrm{~g}$ (67.0\%); Recrytallization solvent : Ethanol; IR $(\mathrm{KBr}) v$ in $\mathrm{cm}^{-1}$ : $3285(\mathrm{NH}), 3065$ (C-H aromatic ring), 2949 (C-H aliphatic), $1725(\mathrm{C}=\mathrm{O}), 1564(\mathrm{C}-----\mathrm{C}$ of aromatic ring), $1167(\mathrm{C}-\mathrm{N}), 1075$ (C-O-C), 1055 (N-N), 675 (C-S-C). ${ }^{1} \mathrm{H}-\mathrm{NMR}$ (DMSO-d $\left.d_{6}\right) \delta 8.14$ (d, $1 \mathrm{H}, \mathrm{CH}-\mathrm{A} r, J=11.0 \mathrm{~Hz}), 7.90\left(\mathrm{~s}, 1 \mathrm{H}, H_{3}\right.$ of quinoline), 7.81 (d, $1 \mathrm{H}, H_{5}$ of quinoline, $\left.J=6.0 \mathrm{~Hz}\right), 7.78\left(\mathrm{~d}, 1 \mathrm{H}, H_{7}\right.$ of quinoline, $J=9.0 \mathrm{~Hz}$ ), $7.62\left(\mathrm{t}, 1 \mathrm{H}, H_{6}\right.$ of quinoline, $\left.J=7.5 \mathrm{~Hz}\right), 7.21$ $7.38(\mathrm{~m}, 4 \mathrm{H}, \mathrm{Ar}-H), 6.63\left(\mathrm{~s}, 1 \mathrm{H}, \mathrm{N} H\right.$, exchangeable with $\left.\mathrm{D}_{2} \mathrm{O}\right)$, 3.71 (s, $2 \mathrm{H}, \mathrm{CH}_{2}$ of thiazolidinone), $3.54\left(\mathrm{~s}, 3 \mathrm{H}, \mathrm{OCH}_{3}\right), 3.19$ (s, 3H, $\left.\mathrm{OCH}_{3}\right), 2.75\left(\mathrm{~s}, 3 \mathrm{H}, \mathrm{CH}_{3}\right)$.MS: $[\mathrm{M}]^{+}$at $\mathrm{m} / z$ 395. Anal. calcd for $\mathrm{C}_{21} \mathrm{H}_{21} \mathrm{~N}_{3} \mathrm{O}_{3} \mathrm{~S}: \mathrm{C}, 63.80 ; \mathrm{H}, 5.32 ; \mathrm{N}, 10.63$; Found: C, 63.93; H, 5.40; N, 10.51.

8-Methoxy-4-methyl-2-amino-[2'-(furfuryl)-4'-oxo-1',3'thiazolidin-3'-yl]quinoline 17: Reagents: compound 7 (1.5 g, $0.01 \mathrm{~mol})$, thioglycolic acid $(0.37 \mathrm{~mL}, 0.01 \mathrm{~mol})$, zinc chloride ( $2.0 \mathrm{~g})$, dioxane $(60 \mathrm{~mL})$; $\mathrm{mp} 183{ }^{\circ} \mathrm{C}$; yield: $0.89 \mathrm{~g}(59.0 \%)$; Recrytallization solvent : Acetone; IR $(\mathrm{KBr}) v$ in $\mathrm{cm}^{-1}: 3275$ (NH), 3075 (C-H aromatic ring), 2951 (C-H aliphatic), 1742 $(\mathrm{C}=\mathrm{O}), 1562$ (C-----C of aromatic ring), $1171(\mathrm{C}-\mathrm{N}), 1055$ 
(C-O-C), 1035 (N-N), 641 (C-S-C). ${ }^{1} \mathrm{H}-\mathrm{NMR}\left(\mathrm{CDCl}_{3}\right) \delta 8.12$ $(\mathrm{d}, 1 \mathrm{H}, \mathrm{CH}-\mathrm{Ar}, J=11.0 \mathrm{~Hz}), 7.91\left(\mathrm{~s}, 1 \mathrm{H}, H_{3}\right.$ of quinoline), $7.83\left(\mathrm{~d}, 1 \mathrm{H}, H_{5}\right.$ of quinoline, $\left.J=6.0 \mathrm{~Hz}\right), 7.73\left(\mathrm{~d}, 1 \mathrm{H}, H_{7}\right.$ of quinoline, $J=9.0 \mathrm{~Hz}), 7.63\left(\mathrm{t}, 1 \mathrm{H}, H_{6}\right.$ of quinoline, $J=7.5 \mathrm{~Hz}$ ), 7.13-7.32 (m, 3H, Ar- $H), 6.65$ (s, $1 \mathrm{H}, \mathrm{N} H$, exchangeable with $\left.\mathrm{D}_{2} \mathrm{O}\right), 3.73\left(\mathrm{~s}, 2 \mathrm{H}, \mathrm{CH}_{2}\right.$ of thiazolidinone), $3.55\left(\mathrm{~s}, 3 \mathrm{H}, \mathrm{OCH}_{3}\right)$, 2.76 (s, 3H, $\mathrm{CH}_{3}$ ). MS: $[\mathrm{M}]^{+}$at $\mathrm{m} / z$ 355. Anal. calcd for $\mathrm{C}_{18} \mathrm{H}_{17}$, $\mathrm{N}_{3} \mathrm{O}_{3} \mathrm{~S}$ : C, 60.85; H, 4.79; N, 11.83; Found: C, 60.91; H, 4.69; $\mathrm{N}, 11.91$.

\section{Biological Evaluation}

Antibacterial activity. The newly synthesized compounds 3-17 and reference drug, amphicillin, were screened for antibacterial activity against different bacterial strains (gram positive bacteria: $S$. aureus, B. subtilis, and S. epidermis and gram negative bacteria: E. coli, K. pneumoniae and P. aeruginosa) at a concentration of $250 \mu \mathrm{g} / \mathrm{mL}$ by filter paper disc method. ${ }^{15}$ DMSO served as control and due this there was no visible change in bacterial growth. The discs of Whatmann filter paper were prepared with standard size $(7.0 \mathrm{~mm})$ and kept into $1.0 \mathrm{Oz}$ screw capped wide mouthed containers for sterilization. These bottles are kept in to hot air oven at a temperature of $150{ }^{\circ} \mathrm{C}$. Then, the prepared solutions of test compounds and standard drug (dissolved in DMSO) of desired concentration were poured into their respective bottles. Further, the discs are transferred to the inoculated plates with a pair of fine pointed tweezers. To prevent contamination tweezers may be kept with their tips in $70 \%$ alcohol and flamed off before used. Before using the test organisms, which were grown on nutrient agar, they were sub-cultured in nutrient broth at a temperature of $37^{\circ} \mathrm{C}$ for $18-20 \mathrm{~h}$. Each disc was applied carefully to the surface of agar without lateral movement once the surface had been touched. Now, the plates were incubated for $24 \mathrm{~h}$ at a temperature of $37^{\circ} \mathrm{C}$. Care was taken not to stockpile the plates. Clear zones of inhibition in millimeters have indicated the relative susceptibility of the bacteria to the compounds 3-17 and amphicillin, standard.

Minimal inhibitory concentration (MIC). The antimicrobial activity was assayed in vitro by the two fold broth dilution ${ }^{16}$ against different bacterial strains (gram positive bacteria: $S$. aureus, B. subtilis, and S. epidermis and gram negative bacteria: E. coli, $K$. pneumoniae and $P$. aeruginosa). The minimal inhibitory concentrations ( $\mathrm{MIC}, \mu \mathrm{g} / \mathrm{mL}$ ) were defined as the lowest concentrations of compound that completely inhibited the growth of the bacterial strain. All compounds dissolved in dimethylsulfoxide (DMSO) were added to culture the media. Mueller hinton broth for bacteria to obtain final concentrations ranging from $100 \mu \mathrm{g} / \mathrm{mL}$ to $0.781 \mu \mathrm{g} / \mathrm{mL}$. The amount of DMSO was never exceeded from $1 \% \mathrm{v} / \mathrm{v}$. Inocula was consisted of $5.0 \times$ $10^{4}$ bacteria $/ \mathrm{mL}$. The MICs were noted after incubation at a temperature of $37{ }^{\circ} \mathrm{C}$ for $24 \mathrm{~h}$. Media and media with $1 \% \mathrm{v} / \mathrm{v}$ DMSO were employed as growth controls, and amphicillin was used as a standard drug.

Acknowledgments. The authors are thankful to Sophisticated Analytical Instrument Facility, Indian Institute of Technology Madras, Chennai, India for spectral and elemental analysis and Head, Department of Microbiology, L. L. R. M. Medical College, Meerut, India for antifungal and antibacterial activities. This paper is the part of Ph.D. thesis of Sheoraj Singh.

The authors have declared no conflict of interest.

\section{References}

1. Appelbaum, P. C.; Hunter, P. A. Int. J. Antimicro. Ag. 2008, 5, 16.

2. Abdel-Moty, S. G.; Abdel-Rahman, M. H.; Elsherief, H. A.; Kafafy, A. H. N. Bull. Pharm. Sci. 2005, $28,79$.

3. Vlahov, R.; Parushev, St.; Vlahov, J.; Nickel, P.; Snatzke, G. Pure Appl. Chem. 1990, 62, 1303.

4. Normand-Bayle, M.; Bénard, C.; Zouhiri, V.; Mouscadet, J.; Leh, H.; Thomas, C.; Mbemba, G.; Desmaële, D.; d'Angelo, J. Bioorg. Med. Chem. Lett. 2005, 15, 4019.

5. Hazeldine, S. T.; Polin, L.; Kushner, J.; White, K.; Corbett, T. H.; Biehl, J.; Horwitz, J. P. Bioorg. Med. Chem. 2005, 13, 1069.

6. He, J.; Yun, L.; Yang, R.; Xiao, Z.; Cheng, J.; Zhou, W.; Zhang, Y. Bioorg. Med. Chem. Lett. 2005, 15, 2980.

7. Dardari, Z.; Lemrani, M.; Bahloul, A.; Sebban, A.; Hassar, M.; Kitane, S.; Berrada, M.; Boudouma, M. Farmaco 2004, 59, 195.

8. Savini, L.; Chiasserini, L.; Pellerano, C.; Filippelli, W.; Falcone, G. Farmaco 2001, 56, 939.

9. Bonsignore, L.; De Logu A.; Loy, G.; Lavagna, S. M.; Secci, D. Eur. J. Med. Chem. 1994, 29, 479.

10. Raman, N.; Thalamuthu, S.; Dhaveethu, J.; Raja, H.; Neelakandan, M. A.; Banerjee, S. J. Chil. Chem. Soc. 2008, 53, 145.

11. Singh, T.; Sharma, S.; Srivastava, V. K.; Kumar, A. Indian J. Chem. 2006, $45 B, 1557$

12. Sharma, P.; Kumar, A.; Sharma, S. Indian J. Chem. 2004, 43B, 585.

13. Vicini, P.; Geronikaki, A.; Incerti, M.; Zani, F.; Dearden, J.; Hewitt, M. Bioorg. Med. Chem. 2008, 16, 3714.

14. Kumar, V.; Singh, S.; Sharma, S. K.; Kumar, A.; Sharma, S. Arch. Pharm. Chem. Life Sci. 2002, 79, 768.

15. Gould, J. C.; Bowie, J. H. Edi. Med. J. 1952, 59, 178.

16. Jorgersen, J. H.; Turnidge, J. D.; Washington, J. A. Manual of Clinical Microbiology; Murray, P. R., Baron, E. J., Pfaller, M. A., Tenover, F. C., Nolken, R. H., Eds.; American Society for Microbiology: Washington, DC, 1999; p 1275. 